Lerussi, R y Scocozza, R.D. Elaboraciones jurisprudenciales en torno a la Compensación Económica en Argentina Derecho y Ciencias Sociales. Octubre 2018. Nº Pgs 93-112 ISNN 1852-2971. Instituto de Cultura Jurídica

\title{
Elaboraciones jurisprudenciales en torno a la Compensación Económica en Argentina
}

Case studies on economic compensation after two years of the new Civil and Commercial Code in Argentina

\section{Romina Lerussi y Romina Daniela Scocozza.}

\section{Resumen:}

El nuevo Código Civil y Comercial de la Nación Argentina entró en vigencia en agosto de 2015 e incorporó importantes modificaciones en materia de divorcio y cese de uniones convivenciales. Entre las innovaciones más destacadas encontramos la figura de la compensación económica tras la disolución del matrimonio o del cese de la unión convivencial (artículos 441 y 524, respectivamente). Desde la sanción del nuevo Código se han generado debates y preguntas en torno a este instituto, tanto en la práctica legal privada, como en la labor judicial y doctrinaria. En el presente artículo presentamos, a partir de material jurisprudencial, dos aspectos que consideramos relevantes para abordar esta figura. Los mismos son una brújula que permite hacer un primer balance de la misma a poco más de dos años de vigencia del nuevo Código. Los aspectos son: principios del derecho involucrados en este instituto, y su cuantificación.

Palabras Clave: Compensación económica ; principios; cuantificación ;Argentina

\begin{abstract}
:
The new Civil and Commercial Code of Argentina came into force in August 2015 and had incorporated important changes in matters of divorce and the cessation of the convivial union. Among the legal innovations, we find particularly interesting the figure of economic compensation after the dissolution of marriage, or the end of the convivial union (articles 441 and 524, respectively). Since the adoption of the new Code, debates and questions have been generated around this institute, both in legal private practices, as well as in judicial work and doctrine. In this article and from jurisprudential material, we present two aspects that we consider relevant to approach this figure and that allow -as a compass- to make a first balance. The two are: principles of law involved in this institute, and its quantification.
\end{abstract}

Keywords: Economic compensation ; principles ; quantification ; Argentina

\footnotetext{
- Romina Lerussi: Doctora en Ciencias Sociales (UBA). Investigadora Asistente (FemGeS-CIFFyH-UNC / CONICET) rclerussi77@gmail.com Romina Daniela Scocozza: Abogada (UNC) Litigante en los Fueros Civil, Laboral y Familia. Diplomada en Desarrollo Humano con Perspectiva de Género y Derechos Humanos (UNCINECIP). Investigadora asistente (CIJS-SECyT, Córdoba).romyscocozza@gmail.com
} 
Lerussi, R y Scocozza, R.D. Elaboraciones jurisprudenciales en torno a la Compensación Económica en Argentina Derecho y Ciencias Sociales. Octubre 2018. Nº Pgs 93-112 ISNN 1852-2971. Instituto de Cultura Jurídica

\section{Elaboraciones jurisprudenciales en torno a la Compensación Económica a dos años de vigencia del Código Civil y Comercial Argentino.}

\section{Romina Lerussi y Romina Daniela Scocozza}

\section{Presentación}

El nuevo Código Civil y Comercial de la Nación Argentina (en adelante CCC) ${ }^{1}$, que entró en vigencia en agosto de 2015, incorporó importantes modificaciones en materia de divorcio y cese de uniones convivenciales. Entre las innovaciones más destacadas encontramos la figura de la compensación económica tras la disolución del matrimonio por divorcio o por su nulidad (en este último caso, siempre que el cónyuge que reclama sea de buena fe), o por el cese de la unión convivencial (arts. 441, 428, 429 y 524, respectivamente). ${ }^{2}$

Desde el punto de vista del nuevo CCC, frente a un procedimiento de divorcio, el art. 441 dispone: "[e]l cónyuge a quien el divorcio produce un desequilibrio manifiesto que signifique un empeoramiento de su situación y que tiene por causa adecuada el vínculo matrimonial y su ruptura, tiene derecho a una compensación. Esta puede consistir en una prestación única, en una renta por tiempo determinado o, excepcionalmente, por plazo indeterminado". La nueva regulación civil establece que esta institución reconoce dos fuentes posibles. La primera es el acuerdo de partes como fruto de la expresión libre y voluntaria de la autonomía de los ex miembros de la unión matrimonial o convivencial. Para el caso en que no exista tal acuerdo, sea por la procedencia misma de la solicitud, por el monto solicitado o por la modalidad de pago, el CCC se encarga de establecer ciertas pautas que los/las operadores jurídicos deben tener en cuenta para su fijación vía judicial. Entre otras, destacamos la dedicación que cada cónyuge/conviviente brindó a la familia y a la crianza y educación de hijos/as, la capacitación laboral y la posibilidad de acceder a un empleo del cónyuge/conviviente que solicita la compensación económica (art. 442). Al respecto, entendemos que las pautas enumeradas lo son al sólo efecto ejemplificativo o enumerativo. La norma no establece el carácter taxativo de las circunstancias enumeradas, sino que dispone que las mismas deberán ser valoradas entre otras y que, agregamos, podrían darse en el caso concreto. Por lo que un correcto entendimiento de este artículo, interpretado sistemáticamente y en consonancia con los principios receptados para los procesos de familia en los arts. 706 del CCC y siguientes, no dejan lugar a una conclusión diferente.

\footnotetext{
${ }^{1}$ Ley $\mathrm{N}^{\circ} 26.994$, de 2014.

${ }^{2}$ A lo largo del presente artículo usamos indistintamente el término compensación económica o su abreviación CE.
} 
Lerussi, R y Scocozza, R.D. Elaboraciones jurisprudenciales en torno a la Compensación Económica en Argentina Derecho y Ciencias Sociales. Octubre 2018. N Pgs 93-112 ISNN 1852-2971. Instituto de Cultura Jurídica

Por su parte, en caso de cese de la unión convivencial, el art. 524 indica: "Cesada la convivencia, el conviviente que sufre un desequilibrio manifiesto que signifique un empeoramiento de su situación económica con causa adecuada en la convivencia y su ruptura, tiene derecho a una compensación. Esta puede consistir en una prestación única o en una renta por un tiempo determinado que no puede ser mayor a la duración de la unión convivencial". Si los convivientes no pactan el derecho a la compensación económica a favor de uno/a, el art. 525 establece que la pueden solicitar judicialmente. A su vez, se debe determinar la procedencia y el monto de la CE sobre la base de diversas circunstancias indicadas en la norma.

Desde la sanción del nuevo CCC, se han generado debates y preguntas en torno a este instituto, tanto en la práctica legal privada, como en la labor judicial y doctrinaria. En el presente artículo presentamos, a partir de material jurisprudencial, dos aspectos que consideramos relevantes para abordar esta figura y que entendemos son una brújula que permite hacer un primer balance de la misma. Los dos aspectos son: principios del derecho involucrados en la CE y, una vez determinada su procedencia, posibles parámetros para su cuantificación.

A partir de este esquema, organizamos el texto del siguiente modo. En primer lugar y teniendo como material de análisis jurisprudencial específicamente un fallo emanado de la Cámara de Apelación en lo Civil y Comercial de la ciudad de Junín (Buenos Aires, Argentina) del año 2016, presentamos los que creemos son los dos principios fundantes de la figura de la $\mathrm{CE}$, a saber: el principio de igualdad, conectado con las que entendemos son algunas aristas de la denominada ética de los/las vulnerables (Highton, 2015), y el principio de solidaridad familiar bajo el influjo de esta nueva figura. Vale decir que hemos seleccionado dicho fallo para abordar este punto, por considerar el contenido de su argumentación como un primer precedente novedoso en la jurisprudencia argentina en la materia.

En segundo lugar y para desplegar algunas inquietudes y respuestas en torno a la medición de la CE, analizamos un fallo del año 2017 dictado por el Juzgado de Familia de Paso de los Libres (provincia de Corrientes, República Argentina) en donde luego del divorcio, una de las partes promueve incidente solicitando la fijación de una CE a su favor. Hemos tomado este fallo por su actualidad en el tratamiento del instituto y por articular algunos aspectos relevantes vinculados a la procedencia y cuantificación de la CE y que, a su vez, conectamos con respuestas dadas en otros fallos. 
Lerussi, R y Scocozza, R.D. Elaboraciones jurisprudenciales en torno a la Compensación Económica en Argentina Derecho y Ciencias Sociales. Octubre 2018. Nº Pgs 93-112 ISNN 1852-2971. Instituto de Cultura Jurídica

En las conclusiones, reconstruimos los aspectos centrales del artículo y esbozamos algunos interrogantes y desafíos para la labor jurídica. A nuestro entender esta nueva figura tiene implicancias en todo el sistema jurídico que habrá que analizar y revisar punto por punto y en su conjunto. En este desafío se inscribe el presente texto.

\section{Principios}

A continuación analizamos los que creemos son los dos principios fundantes de la figura de la CE. Tal como indicáramos, lo hacemos a partir de un fallo emanado de la Cámara de Apelación en lo Civil y Comercial de la ciudad de Junín (Buenos Aires, Argentina) del año 2016. En primer lugar, describimos brevemente el caso. Luego, desplegamos por un lado el principio de igualdad que conectamos con la indicación dada en los Fundamentos del Anteproyecto del CCC (2014) referida a la ética de los/las vulnerables. En palabras de Highton (2015): "El Código [nuevo] busca la igualdad real y desarrolla una serie de normas orientadas a plasmar una verdadera ética de los vulnerables" (p.5). Por el otro lado, el principio de solidaridad familiar bajo el efecto de esta figura. Como esquema de presentación, exponemos los argumentos centrales del fallo que guardan relación con cada principio, para luego introducir algunos comentarios.

Dos aclaraciones generales del apartado. Primera, nos atenemos a los aspectos del fallo que entendemos responden al objetivo del presente punto, dejando fuera del análisis aquellos que lo exceden. Segunda, analizamos los dos principios involucrados con el material argumental del fallo, a partir del cual introducimos algunas consideraciones desde una lectura socio jurídica. No es nuestra intención exponer el conjunto de debates y literatura jurídica relativa a cada principio, sino más bien su carácter orientativo aplicado a la figura de la $\mathrm{CE}$.

\section{2.a. El caso}

El día 25 de octubre del año 2016 en la ciudad de Junín (provincia de Buenos Aires, Argentina), la Cámara de Apelación en lo Civil y Comercial de dicha ciudad dictó sentencia sobre la causa Expte. No JU-7276-2012 caratulada: “G., M. A. C/ D. F., J. M. S/ALIMENTOS", pronunciándose sobre nuevos aspectos vinculados al instituto de la compensación económica con motivo, en el caso, de un divorcio. ${ }^{3}$

\footnotetext{
${ }^{3}$ Excma. Cámara Nacional de Apelación en lo Civil y Comercial de Junín, $\mathrm{N}^{\circ}$ de orden 184, libro de sentencia No 57, (25/10/2016), en autos «G., M. A. c/ D. F., J. M. s/ alimentos». Aporte del órgano emisor. Las citas textuales tomadas del fallo e indicadas entre comillas corresponden a la versión de la sentencia mimeografiada que referimos del siguiente modo: CámApCyCom Junín, 2016.
} 
Lerussi, R y Scocozza, R.D. Elaboraciones jurisprudenciales en torno a la Compensación Económica en Argentina Derecho y Ciencias Sociales. Octubre 2018. Nº Pgs 93-112 ISNN 1852-2971. Instituto de Cultura Jurídica

En la primera instancia, el Juez titular del Juzgado Civil y Comercial $\mathrm{n}^{\mathrm{o}} 2$, consideró que el cese de la convivencia ocasionó a la parte afectada (Sra. M. A. G.) un abrupto descenso en su nivel de vida, juzgando equitativo fijar en su favor una compensación económica bajo la modalidad de renta mensual, sin fijación de plazo y con efecto retroactivo al día de la presentación de la demanda. ${ }^{4}$

Dicha sentencia fue apelada y la Cámara decidió "fijar la compensación económica en favor de la actora y a cargo de su ex cónyuge Sr. D. F. en la suma única de pesos ciento cincuenta mil \$ 150.000” (CámApCyCom Junín, 2016).

En la sentencia se brindan elementos que consideramos orientadores, a partir de citación de doctrina así como de la labor argumental desplegada por la propia Cámara, en especial aquellos referidos a la plataforma de principios centrales de este instituto.

\section{2.b. Principio de igualdad}

En la Argentina, la igualdad, considerada tanto en su faz formal como material, es un principio constitucional (artículo 16, 37, 75 inc. 23 Constitución Nacional de la República Argentina - CN) y convencional (artículo 2, incisos b y c y artículo16, CEDAW). ${ }^{5}$ Estos principios atraviesan la regulación del matrimonio y de la unión convivencial, teniendo un especial impacto en los efectos de una eventual ruptura, en el marco de la progresiva constitucionalización del derecho privado. Este proceso alude a la tendencia en este país a la aplicación directa e inmediata de la Constitución Nacional y de los Tratados Internacionales de Derechos Humanos en el derecho de familia (art. 75, inc. $22 \mathrm{CN}$ ). Este es el marco general en donde se sitúa la figura de la $\mathrm{CE}$ y es en consonancia con ello que la Cámara cuyo fallo analizamos, construye la argumentación para dictar la nueva sentencia.

En primer lugar, en el fallo se indica que:

La compensación económica constituye una valiosa herramienta proactiva para lograr una mayor igualdad real, no solo formal, como pretende el Código en todo su articulado, tomándose como eje la protección al más vulnerable o débil. La recepción de esta figura contribuye a que el cónyuge que sufrió un menoscabo económico pueda lograr su independencia económica hacia el futuro, evitando recurrir al pago de alimentos para poder rehacer su vida. (Herrera et al, 2014 citada en CámApCyCom Junín, 2016).

\footnotetext{
${ }^{4}$ Fs. 357/363, Juzgado Civil y Comercial no 2 .

${ }^{5}$ CEDAW: En castellano refiere a la Convención sobre la eliminación de todas las formas de discriminación contra la mujer.
} 
Lerussi, R y Scocozza, R.D. Elaboraciones jurisprudenciales en torno a la Compensación Económica en Argentina Derecho y Ciencias Sociales. Octubre 2018. Nº Pgs 93-112 ISNN 1852-2971. Instituto de Cultura Jurídica

Es decir, tres elementos son sustanciales para que proceda la solicitud de CE, a saber: la existencia de un manifiesto desequilibrio económico provocado por la ruptura del vínculo, en este caso matrimonial, que se traduzca en un menoscabo económico tal que no permita que la parte afectada pueda rehacer su vida a futuro de manera más o menos equilibrada. De lo que se infiere, demostrado lo anterior, una situación fáctica de desigualdad (de allí, la afectación a este derecho) provocada por la ruptura del vínculo en donde una de las partes, frente al divorcio, queda en una clara situación de vulnerabilidad. Dicho en otros términos, frente a la ruptura del plan de vida existencial (Pellegrini, 2014) o de la comunidad de vida (Solari, 2014) y a partir de los roles y las funciones desempeñados durante la vida en común, una de las partes queda en clara situación de desventaja económica. En palabras de Pellegrini (2014), la figura de la CE: "se erige como una herramienta legal que posibilita y favorece una cierta igualdad real en las condiciones y oportunidades en que cada cónyuge se encuentra una vez finalizado su proyecto de vida en común, condiciones que se vieron afectadas y desbalanceadas justamente a causa de dicho quiebre" (p. 459). Tanto casuísticamente en la Argentina cuanto en la literatura comparada, por ejemplo la española (Belio Pascual, 2013), se observa que esto ocurre muy especialmente a raíz de la dedicación no remunerada de una de las partes -en parejas heterosexuales mayoritariamente la mujer-, al hogar, al trabajo de cuidados y a las tareas domésticas.

En definitiva, siguiendo el tono de los Fundamentos del Anteproyecto del CCC, el instituto se aleja de todo contenido asistencial por un lado y, por el otro, coloca la mirada en la función protectoria (CámApCyCom Junín, 2016; Pellegrini, 2014), a lo que podríamos agregar y por lo dicho anteriormente, su función garantista de los derechos económicos y patrimoniales en especial de las mujeres.

Ahora bien, de la regulación reseñada surgen numerosas preguntas. Qué es lo que se compensa, a qué refiere la expresión compensar las situaciones de desigualdad real y qué indica la orientación derivada de los Fundamentos del Anteproyecto del CCC referida a la ética de los/las vulnerables aplicada a esta figura. A continuación abordamos estos tres interrogantes a partir de argumentos del propio fallo y aportando elementos socio jurídicos que puedan enriquecer el debate sobre este principio referido a la CE.

En los argumentos del juez de primera instancia y que pueden leerse en la sentencia apelada, hay un elemento importante que la Cámara despeja desde el punto de vista del interrogante acerca de qué es lo que se compensa. Leemos en el fallo que en la primera instancia el juez evalúa que: "la ruptura del vínculo matrimonial provocó en la cónyuge reclamante un desequilibrio en su patrimonio, al verse privada de los ingresos que aportaba su ex cónyuge 
Lerussi, R y Scocozza, R.D. Elaboraciones jurisprudenciales en torno a la Compensación Económica en Argentina Derecho y Ciencias Sociales. Octubre 2018. Nº Pgs 93-112 ISNN 1852-2971. Instituto de Cultura Jurídica

y reemplazado por los alimentos provisorios fijados en el juicio de divorcio, juzgando que la cuota fijada por dicho concepto no alcanzaba a cubrir los requerimientos necesarios para mantener el nivel de vida anterior a la ruptura matrimonial” (CámApCyCom Junín, 2016) ${ }^{6}$.

Como primera indicación, la compensación no tiene como fin el procurar que la parte afectada por el desequilibrio económico a raíz de la ruptura pueda mantener el nivel de vida anterior. El instituto de la $\mathrm{CE}$, recordamos, tiende a equilibrar una situación que se desencadenó en el matrimonio y como resultado de acuerdos y pactos de la pareja de órdenes diversos, muchas veces tácitos o implícitos y por lo tanto naturalizados e incluso, invisibilizados. Situación, vale decir, que se hace visible con el quiebre del proyecto de vida en común. En palabras de la propia Cámara: "Lo equitativo y razonable no es aquí la búsqueda de una nivelación o igualación patrimonial entre las partes sino la recomposición del correspondiente a uno de ellos por el empobrecimiento - generalmente por la frustración o postergación del crecimiento propio, pérdida de chances u oportunidades y ayudas que hubiere brindado- a la par y vinculado al enriquecimiento del otro, durante la convivencia" (CámApCyCom Junín, 2016).

En tal sentido, tal como lo indica la Cámara, la CE procura equilibrar una situación de desigualdad, a lo que agregamos que ya estaba instalada en la forma en la que se había configurado el matrimonio. Este es el aspecto que nos parece central y en el que la Cámara no acentúa su argumentación o al menos no de este modo. Es precisamente a esa situación a la que refiere, a nuestro entender, la expresión compensar las situaciones de desigualdad real.

La figura de la CE nos permite ver lo que estuvo oculto a lo largo del pacto matrimonial (en este caso). Es decir, ver precisamente ese estado de desigualdad real en donde una de las partes dedicó total o parcialmente su tiempo personal a la vida en común, mientras que la otra dedicó total o parcialmente su tiempo personal al desarrollo del proyecto individual, independientemente de la aportación dineraria necesaria para la manutención de la vida en común. Es ese capital individual acumulado (por ejercicio y desarrollo de profesión, oficio, comercio, industria, actividad laboral) el que, dada la ruptura de la comunidad, se hace visible. ${ }^{7}$ Lo que se intenta compensar es justamente la situación en la que queda la parte vulnerada: años de trabajo no remunerado para la comunidad familiar equivalente a años de

\footnotetext{
${ }^{7}$ Incluso cuando hay contratación (total o parcial), la negociación y demás aspectos de la misma en general es entre mujeres: la empleadora y la trabajadora de casas particulares. Vale indicar que el sector en casas particulares en la Argentina se compone aproximadamente de casi un millón de trabajadores/as, integrado en un $95 \%$ por mujeres (El Hay, 2013) y registrado sólo en un 15\% (con un 85\% de informalidad) (Ojeda, 2013).
} 
Lerussi, R y Scocozza, R.D. Elaboraciones jurisprudenciales en torno a la Compensación Económica en Argentina Derecho y Ciencias Sociales. Octubre 2018. Nº Pgs 93-112 ISNN 1852-2971. Instituto de Cultura Jurídica

no desarrollo de proyecto personal para, quebrado el vínculo, enfrentar una nueva vida. Desigualdad de oportunidades, dirá la Cámara, para afrontar la vida en forma independiente después de la ruptura, situación que puede haber: "aniquilado [total o parcialmente] cualquier expectativa de abrirse camino económicamente en forma independiente, quedando sumidas en una situación totalmente desfavorable de no ver reconocida con carácter indefinido una compensación por sus renuncias" (CámApCyCom Junín, 2016). En definitiva, y esta es una novedad argumental que introduce el tribunal: "se trata de un instituto que tiene la mirada puesta en el futuro, en cuanto tiende a reequilibrar a los miembros de la pareja, con el fin de ponerlos en condiciones de proyectarse económicamente" (Ibíd.)

Finalmente, respecto al interrogante acerca la ética de los/las vulnerables aplicada a esta figura, hacemos algunas consideraciones. El término vulnerabilidad es ambiguo y se presta a un sinnúmero de usos, de los cuales indicamos tres. En primer lugar, se usa para hacer referencia a situaciones de vulnerabilidad individual, por ejemplo, nacer con una discapacidad total o parcial, o un estado de enfermedad inhabilitante o de vejez no saludable (enfermedades típicas como el Alzheimer), son situaciones de vulnerabilidad individual en principio no producidas (aunque sí afectadas) por el contexto. En segundo lugar, se usa para hacer referencia a situaciones de vulnerabilidad en el sentido de que las personas somos vulnerables (cuando nacemos, por enfermedad, vejez, cambios climáticos, alimentarios, etcétera) y por lo tanto, necesitadas de cuidados a lo largo de la vida. Es decir, cuidados en un sentido dado por el paradigma de los cuidados dentro de las investigaciones y respuestas referidas a este asunto que dan la economía feminista crítica y algunas líneas dentro de los feminismos jurídicos (Pérez Orozco, 2006; Pautassi, 2007). En tercer lugar, se usa para conectar la vulnerabilidad con situaciones de violación de derechos fundamentales. Situaciones en las que se nace (por contexto de vida) o a las que se arriba, por trayectoria personal y/o laboral. Este último constituye el uso habitual que se hace de este término cuando se habla de sectores vulnerables o grupos vulnerables en la legislación, las políticas públicas y las acciones sociales y comunitarias. Este es, creemos, el sentido que se podría designar a la expresión ética de los vulnerables indicada en los fundamentos del Anteproyecto del CCC (2014), remarcada por Highton (2015) y que incide en la configuración del instituto en estudio.

En fin, frente a la solicitud de la CE, mayoritariamente ejercida por mujeres, no es que las vulnerables sean las mujeres per se (que es para quienes especialmente, entendemos, está pensado este instituto). Los pactos matrimoniales o convivenciales en sistemas 
Lerussi, R y Scocozza, R.D. Elaboraciones jurisprudenciales en torno a la Compensación Económica en Argentina Derecho y Ciencias Sociales. Octubre 2018. N Pgs 93-112 ISNN 1852-2971. Instituto de Cultura Jurídica

heteronormativos jerarquizados, suponen pactos sexuales que incluyen la división sexual del trabajo (y esto lo recoge el derecho en todo el sistema). Es decir, roles jerarquizados y funciones estereotipadas en las que cada una de las partes ya tiene pre-asignado su lugar. Todo lo cual puede o no darse de hecho. Ahora bien, es precisamente frente a la ruptura de la comunidad de vida que puede visibilizarse dicho esquema, bajo la forma de un estado de manifiesto desequilibrio económico de una de las partes (en general, la mujer) para iniciar una nueva vida. Situación que, repetimos, no es sino efecto de un estado de desigualdad real progresivo creado a lo largo del matrimonio. De allí el estado de vulnerabilidad estructural que hay que atender y revertir no sólo vía todo el sistema jurídico sino en la sociedad en su conjunto.

\section{2. c. Principio de solidaridad familiar}

En referencia a los Fundamentos del Anteproyecto de Código Civil y Comercial de la Nación Argentina (2014) recogidos en el nuevo CCC, la CE se basa en el principio de solidaridad familiar y en que el matrimonio o la convivencia no sean causa fuente de enriquecimiento o empobrecimiento económico de un cónyuge o conviviente a costa del otro (Herrera et al, 2014). Luego, como bien indica la Cámara del fallo en análisis, eso no implica imponer la igualdad absoluta entre los ex cónyuges o ex convivientes (tema que tratamos en el punto anterior), sino más bien la CE intenta, dirá la Cámara: “compensar el empobrecimiento económico sufrido por uno con respecto al otro, causado por las renuncias en pos de la asistencia o solidaridad familiar, que en contracara importan la posibilidad de quien no lo hace por el proyecto común de vida de ambos, y se ve favorecido en poder abocarse al desarrollo de su proyecto industrial, comercial, profesional o de vida laboral más allá de la familia, o lo hace en mayor medida que el otro" (CámApCyCom Junín, 2016).

A su vez, siguiendo el tono de los Fundamentos del Anteproyecto del CCC (2014), la CE como indicáramos anteriormente, se aleja no sólo de todo contenido asistencial (eje y principio de otras instituciones, por ejemplo, el deber alimentario), sino además de cualquier imputación referida a un posible incumplimiento de deberes matrimoniales o de la idea de culpa/inocencia en las causas que definieron la ruptura como elemento determinante de su asignación. Al adoptar el nuevo Código el sistema de divorcio incausado, para que proceda la CE debe sólo darse la ruptura del matrimonio (en el caso en estudio), independientemente de las causas del divorcio, y bajo las indicaciones de la norma referidas a la CE ya mencionadas con anterioridad. Así lo argumenta la Cámara: 
Lerussi, R y Scocozza, R.D. Elaboraciones jurisprudenciales en torno a la Compensación Económica en Argentina Derecho y Ciencias Sociales. Octubre 2018. Nº Pgs 93-112 ISNN 1852-2971. Instituto de Cultura Jurídica

La compensación económica es una figura que se incorpora al texto civil y comercial en total consonancia con el régimen de divorcio incausado; a ninguna de las dos figuras legales (compensación y divorcio) les interesa la culpa o inocencia de los cónyuges sino el acaecimiento de ciertas circunstancias que producen un desequilibrio económico que un cónyuge o conviviente debe compensar al otro. En este contexto, si la ruptura matrimonial deprimió al cónyuge que soportó la infidelidad, si dejó el hogar familiar y se fue a vivir a la casa de un amigo, etc. son circunstancias que no interesan para dirimir judicialmente si se hace o no lugar al pedido de compensación económica, o cómo se evalúan en el caso que fueran procedentes porque se dan los requisitos legales. En otras palabras, nada más alejado de la idea de culpa que la compensación económica, dando cuenta de ello las diferentes variables que explicita el art. 442 para su fijación judicial, todas de índole objetiva que no indagan sobre conductas culpables por parte de los cónyuges. (CámApCyCom Junín, 2016).

De allí algunas indicaciones de la Cámara dadas en el fallo. Leemos, como parte de la argumentación de la defensa del demandado (el ex esposo) lo siguiente: "No se encuentran cumplidos los recaudos establecidos en el art. 442 del Cód. Civ. y Com. destacando que siempre acompañó a su esposa en las decisiones relativas a su profesión docente” (Ibíd.). En esta enunciación hay un punto importante a indicar en cuanto a los efectos de la CE sobre el principio de solidaridad familiar. El eje aquí no es que se haya acompañado o no en las decisiones de la otra parte (en este caso, la mujer, ex esposa), ni siquiera es relevante que por mutuo acuerdo hayan decidido que ella se dedique al trabajo de cuidados y doméstico de manera prioritaria y no remunerada. El meollo de la CE es que dada la conclusión del matrimonio, repetimos, una de las partes queda desamparada desde el punto de vista económico para afrontar una vida futura de manera autónoma vía un proyecto personal. Es más, en el caso en cuestión, el ex esposo (médico) no sólo se observa y se destaca en el fallo que ha incrementado sus ingresos y cargos laborales, sino que además ha elevado su capital simbólico y profesional que es indivisible, es suyo y le permitirá continuar de manera independiente con su vida luego de la ruptura de la comunidad matrimonial. De allí la CE pensada para la parte afectada. En este sentido, entendemos que la CE no sólo es un efecto propio del divorcio como del cese de una unión convivencial o, incluso, de la nulidad matrimonial (Pellegrini, 2014). Conceptual y lógicamente, la CE tiene efectos (retroactivos) sobre el propio principio de solidaridad familiar. 
Lerussi, R y Scocozza, R.D. Elaboraciones jurisprudenciales en torno a la Compensación Económica en Argentina Derecho y Ciencias Sociales. Octubre 2018. Nº Pgs 93-112 ISNN 1852-2971. Instituto de Cultura Jurídica

En este último sentido, hacemos breves comentarios. En primer lugar, entendemos que la figura de la CE tiene implicancias conceptuales directas, decíamos, sobre el concepto de la denominada solidaridad familiar. En el marco del nuevo Código, la solidaridad familiar supone también la valoración por ejemplo de la situación típica del trabajo doméstico y de cuidados no remunerados desde el punto de vista de la organización del tiempo y trabajo personal puesto a disposición para la comunidad de vida, no sólo en miras del presente sino del futuro. Es decir, al momento de su conformación (matrimonio, en el caso en análisis) se presupone una comunidad de mutua colaboración pero al mismo tiempo, se presupone que ambas partes tienen que potenciarse en ese vínculo. Es decir, que ambas partes a través de la unión matrimonial puedan crecer, desarrollarse y progresar no sólo en la comunidad matrimonial sino allí mismo, individualmente. Así, de suspenderse, ambas estén en condiciones de rehacer su vida luego de la ruptura en un marco de cierta igualdad real. De lo que se sigue que de no darse esta situación y en las condiciones indicadas por la norma, una de las partes (incluso habiendo impulsado el divorcio) pueda solicitar la CE como efecto de un derecho vulnerado.

Es oportuno indicar que en este esquema, incluso cuando ambas partes trabajan de manera remunerada, se supone que las labores de cuidados y domésticas están a cargo de la mujer, sea como trabajadora no remunerada o gerenciando la labor de una trabajadora de casa particular contratada. Todo como parte de lo que se entendía, y entiende aún, como el aporte de las mujeres a la comunidad de vida bajo el principio de solidaridad familiar y ayuda mutua, al persistir un sistema de familia patriarcal. ${ }^{8}$ Sumado al hecho fáctico de que el principio de solidaridad familiar deja afuera y por lo tanto oculta (y en esto los tribunales de familia son testigos) las violencias, los conflictos y las desigualdades que existen al interior de las parejas en los procesos de negociación y toma de decisiones (Silbaugh, 1996; Zelizer, 2009).

En fin, la CE es una verdadera novedad legislativa que viene a reconfigurar no sólo la propia institución del matrimonio (y de las uniones convivenciales), sino todo el engranaje dogmático que envuelve al principio de solidaridad familiar.

\section{Procedencia y cuantificación}

\footnotetext{
${ }^{8}$ En el presente artículo entendemos por patriarcado (ejemplo la familia patriarcal) a modalidades de jerarquización de lo humano, en donde un determinado tipo de sujeto-cuerpo-varón-adulto-heterosexual se vuelve relevante en tanto que relación de poder desigual sobre otros sujetos-cuerpos (por ejemplo, las mujeres). Así, en una cultura patriarcal, la configuración social hegemónica que da forma a la cultura legal se sostiene sobre este principio de jerarquía que produce desigualdad y exclusión, por lo tanto violencias. Y, su vez, se articula con otras marcas de jerarquización tales como clase, género, etnia, raza, estatus migratorio.
} 
Lerussi, R y Scocozza, R.D. Elaboraciones jurisprudenciales en torno a la Compensación Económica en Argentina Derecho y Ciencias Sociales. Octubre 2018. Nº Pgs 93-112 ISNN 1852-2971. Instituto de Cultura Jurídica

\section{3.a. Los casos}

La resolución judicial que traemos a estudio fue dictada el 6 de julio de 2017 por el Juzgado de Familia de Paso de los Libres. ${ }^{9}$ Luego del divorcio, la Sra. A. M. L. promovió incidente solicitando la fijación de una CE a su favor. Este pedido fue rechazado por el Sr. J. M. L., quien a su vez interpuso reconvención solicitando a su vez la fijación a su favor de CE.

Se trataba de un matrimonio cuya duración de 22 años tuvo como fruto dos hijas que al momento de la ruptura matrimonial ya habían alcanzado la mayoría de edad. Durante la vigencia del vínculo matrimonial, la vida familiar se organizó en torno a la asignación social estereotípica de roles, en función de la cual en una pareja conformada por personas de distinto sexo el varón desarrolla un rol predominantemente proveedor de recursos económicos-monetarios, realizando trabajos remunerados fuera del hogar. Y, por su parte, la mujer cumple con el cuidado de la casa particular, la crianza y cuidado de las/os hijas/os y, eventualmente, con el cuidado y asistencia de adultos mayores o personas con algún tipo de enfermedad o discapacidad, sea esta permanente o transitoria.

En el caso, el esposo se desempeñó siempre como empleado en relación de dependencia, con ingresos netos de entre 30 y 40 mil pesos mensuales y, al momento de la solicitud de CE, contaba con 30 años de antigüedad (dato importante a los fines previsionales). En tanto que la esposa registraba sólo tres años de trabajo en relación de dependencia (al comienzo del matrimonio) y al momento del divorcio se encontraba empleada en una actividad por la que percibía un ingreso mensual equivalente al $10 \%$ de los haberes que percibía el esposo (poco más de 3000 pesos argentinos y al momento de la sentencia ni un 50\% de lo que equivaldría a un Salario Mínimo Vital y Móvil).

El juzgador entendió que en este punto se configuraba, de cara al futuro de ambos ex cónyuges, el manifiesto desequilibrio que exige la normativa que regula la $\mathrm{CE}$. En el fallo se lee: "el desequilibrio existente entre los medios de vida de cada uno de los ex cónyuges surge palmario, aproximadamente treinta mil pesos mensuales contra aproximadamente tres mil pesos mensuales". Para la determinación de la procedencia, además de las pruebas informativas y documentales aportadas, el tribunal valoró especialmente las declaraciones de testigos que daban cuenta de que a la dedicación de la mujer al hogar, se sumaba un pequeño aporte que lograba de la venta (por las tardes, desde su casa e informalmente) de artículos diversos como discos de música y ropa, cuyos frutos devenían en aportes a la economía

\footnotetext{
9 Juzgado de Familia de Paso de los Libres (provincia de Corrientes, República Argentina), en autos: "Incidente de compensación económica. L., J. A. C/ L., A. M. S/ divorcio" (Expte. I03 13301/02).
} 
Lerussi, R y Scocozza, R.D. Elaboraciones jurisprudenciales en torno a la Compensación Económica en Argentina Derecho y Ciencias Sociales. Octubre 2018. Nº Pgs 93-112 ISNN 1852-2971. Instituto de Cultura Jurídica

familiar. También se valoró que la dedicación a las tareas de cuidado y del hogar excedía lo que estrictamente se puede definir como tareas domésticas. De hecho, en el caso bajo estudio, quedó acreditado que la familia contaba con servicio doméstico contratado, circunstancia que no fue valorada como un factor en contra de la fijación de la CE a cargo del ex esposo y a favor de la ex esposa.

\section{3.b. Dilemas cuantitativos}

Una vez decidido el juzgador a reconocer el derecho de la mujer a obtener una compensación por el desequilibrio económico que le ocasionó la ruptura matrimonial, enfrentó el problema de la fijación de su cuantía. En este punto, la parte hizo una propuesta para definir el modo de calcular la suma dineraria, la cual fue rechazada por el órgano jurisdiccional. La mujer propuso multiplicar el equivalente a un Salario Mínimo Vital y Móvil (SMVM), que al tiempo de la sentencia de divorcio (con fecha 14/10/2016) ascendía a la suma de 6.810 pesos por doce meses, y que el resultado fuera multiplicado por los años que permanecieron unidos en matrimonio. La fórmula propuesta sería la siguiente:

$\$ 6.810 \times 12$ meses $=\$ 81.720$ por año $\times 22$ años de casados $=\$ 1.797 .840$

El monto al que se arribó aplicando este cálculo fue rechazado por el juez y calificado como abultado. Para sostener este calificativo, argumentó que: "no estamos en presencia de una indemnización por pérdida de chances, daños o perjuicios, ni de una cuota alimentaria, sino de una compensación económica que daría la oportunidad a la solicitante de palear el desequilibrio que el divorcio le produjo". A renglón seguido, propuso otro modo de cálculo diferente. El juez tomó también como base el SMVM, pero el vigente a la fecha de su resolución (ya ascendía a la suma de 8.860 pesos, según la modificación vigente desde el 01/07/2017). Lo multiplicó por 12 meses y al resultado obtenido, lo volvió a multiplicar por la cantidad de años laborales que le quedan a la mujer (restando a 65 la edad de la ex esposa que al tiempo de la resolución tenía 47 años). Sobre el resultado, el juzgador calculó el 10\% y fijó este monto como el procedente para la compensación solicitada. La fórmula quedaría así:

$\$ 8.860 \times 12$ meses $=\$ 106.320 \times 18$ años $(65$ años -47 años $)=1.913 .760=10 \%=$

$\$ 191.376$ 
Lerussi, R y Scocozza, R.D. Elaboraciones jurisprudenciales en torno a la Compensación Económica en Argentina Derecho y Ciencias Sociales. Octubre 2018. Nº Pgs 93-112 ISNN 1852-2971. Instituto de Cultura Jurídica

La diferencia entre el valor reclamado (casi dos millones de pesos) y la suma fijada judicialmente (menos de doscientos mil) es, cuanto menos, impresionante. El criterio de la prudencia judicial se combina en este caso con el uso de fórmulas, hasta dar con un monto que, a criterio del juzgador, se aproxima más a un monto adecuado que a uno abultado.

En el caso reseñado hay una solicitud de compensación que especifica claramente las razones para su procedencia. Además, se expresa el monto pretendido y la forma en la que ese monto fue calculado, estipulando los parámetros tenidos en cuenta para ese fin. El demandado rechazó ambos extremos y, ante la falta de acuerdo e incluso en este caso ante la presencia de reconvención, el juzgador es quien debió determinarlos.

En este orden de dilemas, ¿qué ocurre cuando el/la demandante no aclara el monto pretendido sino que se limita a formular la solicitud pero no expresa un monto determinado o determinable sino que deja ese aspecto completamente librado al arbitrio del juez? Es un interrogante interesante que recibió una posible respuesta jurisprudencial a través de la Sala I de la Cámara Nacional de Apelaciones en lo Civil. ${ }^{10}$ En dicho caso fue admitida la posibilidad de que se introduzca el pedido de CE sin precisar el monto solicitado en el escrito que dio inicio a la demanda. El criterio adoptado tanto en primera como en segunda instancia, fue el de rechazar la excepción de defecto legal opuesta por el demandado y confirmar que la no especificación del monto requerido, no poseía una gravedad tal que colocara a éste último en una situación de indefensión.

No podemos compartir el criterio sostenido en estas resoluciones, por los siguientes argumentos. El pedido de CE articulado judicialmente constituye una demanda, más allá de que según el ordenamiento procesal provincial adopte forma incidental. Razón sobrada para entender que debe designarse con precisión qué es lo que está siendo objeto de ese acto procesal, es decir, cuál es la pretensión esgrimida. Suma a este argumento la instancia de conciliación prevista en casi la totalidad de los ordenamientos procesales, instancia que se vería seriamente afectada en sus posibilidades de éxito si el demandado no conoce con exactitud cuánto es lo que se le está demandando. Lo único que puede demandarse es una prestación que puede consistir en una prestación única o en una renta por plazo determinado o, excepcionalmente, indeterminado. ${ }^{11}$ Por todo lo manifestado, resulta violatorio del

\footnotetext{
${ }^{10}$ Cámara Nacional de Apelaciones en lo Civil, Sala I, en autos: "M. L. N. E. c/ D. B., E. A. s/ fijación de compensación" (13/9/2016). La Ley, 7/11/2016 (cita online: AR/JUR/64925/2016, visitada 10/09/2017).

${ }^{11}$ En este sentido, el art. 441 del CCC es claro al disponer que puede pagarse con dinero, con el usufructo de determinados bienes o de cualquier otro modo que acuerden las partes o que determine el juez. Las bastardillas son nuestras.
} 
Lerussi, R y Scocozza, R.D. Elaboraciones jurisprudenciales en torno a la Compensación Económica en Argentina Derecho y Ciencias Sociales. Octubre 2018. Nº Pgs 93-112 ISNN 1852-2971. Instituto de Cultura Jurídica

principio constitucional de defensa en juicio la admisión de un reclamo que no designe con precisión el objeto reclamado.

Las dificultades para la cuantificación no son exclusivas de la CE (Pellegrini, 2017). Cuantificar un pedido que busca una justa recomposición (Anteproyecto CCC, 2014) ante el desequilibrio constatado de posibilidades económicas de los ex cónyuges o ex convivientes conlleva desafíos que, como estamos viendo, no resultan de fácil solución. No obstante, consideramos que no debe eximirse sin más a la parte demandante de realizar el esfuerzo y proponer un monto, los parámetros en los que se haya basado para realizar el cálculo y una modalidad de pago, aunque finalmente lo demandado pueda no ser de recibo de acuerdo con el criterio que el tribunal decida aplicar. ${ }^{12}$

También puede suceder que la suma solicitada y la modalidad de pago obtenga acogimiento en primera instancia pero el criterio sea modificado en instancia de apelación. Tal es la situación del fallo reseñado en el apartado anterior (CámApCyCom Junín, 2016). En este caso ninguno de los juzgadores utiliza la herramienta de las fórmulas. En primera instancia se fija a favor de la actora una renta del $20 \%$ del total facturado mensualmente por el demandado, sin límite temporal y con efecto retroactivo al día de la presentación de la demanda. En segunda instancia, este aspecto es revocado, ya que si bien se mantiene la decisión de fijar una CE a favor de la demandante, la misma es fijada en una suma de pesos ciento cincuenta mil (\$150.000), pagaderos en tres cuotas iguales y consecutivas. Se advierte también en este caso que la objetividad pretendida por el CCC y la igualdad como principio reciben diferentes significaciones según sea aplicada la prudencia judicial.

En fin, en los casos bajo análisis basta con observar que ante la misma plataforma fáctica, los cálculos realizados en primera y segunda instancia modifican significativamente las compensaciones acordadas a las demandantes. Lo importante en este punto es resaltar que

\footnotetext{
12 Para profundizar en aspectos procesales que no abordados en el presente texto, pueden consultarse los siguientes fallos. En referencia a los tiempos de la solicitud de la CE, caducidad y su tratamiento jurisprudencial, véase por un lado, "D. P., R. A. c/ A., M. D. C. - Divorcio vincular - Contencioso", Juzgado de Familia de Segunda Nominación de Córdoba (20/03/2017). Por el otro, en autos "S., A. A. c/P., O. R. s/fijación de compensación arts. 524 y 525, CCCN", Expte 46.075/16, Cámara Nacional de Apelaciones en lo Civil, Sala J (07/10/16), donde se resolvió el rechazo in limine de la pretensión de fijación de CE debido a que había vencido el plazo de caducidad previsto por el art. 442 CCC. En ambos casos, tanto en primera instancia en el caso cordobés como en segunda instancia en la Cámara de Apelaciones, se enfatiza el hecho de que el plazo de seis meses está establecido con tal brevedad con el objetivo de que los cónyuges resuelvan todas las cuestiones patrimoniales que se derivan de la ruptura de manera simultánea al divorcio. Resulta relevante para el ordenamiento procesal de la Provincia de Córdoba que se haya establecido el agotamiento de la etapa pre judicial como requisito de admisibilidad de la demanda de CE, aun cuando se haya introducido dicho pedido en la propuesta de convenio regulador (requisito de admisibilidad de la solicitud de divorcio unilateral, art. 438 CCC) y se haya llevado a cabo la audiencia de conciliación prevista por el mismo artículo.
} 
Lerussi, R y Scocozza, R.D. Elaboraciones jurisprudenciales en torno a la Compensación Económica en Argentina Derecho y Ciencias Sociales. Octubre 2018. Nº Pgs 93-112 ISNN 1852-2971. Instituto de Cultura Jurídica

cualquiera sea el método de cuantificación adoptado por el juez o la jueza, debe cumplirse acabadamente el deber de explicitar la decisión a la que se ha arribado. El camino recorrido, los parámetros tomados en cuenta o dejados de lado en cada caso concreto, eventualmente la o las fórmulas escogidas, incluso, agregamos, las razones por las que el juzgador/a tome la cuantificación ensayada por las partes (o se aparte de ella), deben ser parte de la fundamentación, con ningún otro objetivo más que el de que las partes puedan conocer, controlar y eventualmente controvertir la conclusión obtenida (Acciarri, 2015).

\section{Consideraciones finales}

En el presente artículo nos dedicamos, a partir de material jurisprudencial y reflexión socio jurídica, a abordar los que creemos son dos componentes centrales para pensar y evaluar la figura de la CE a más de dos años de la entrada en vigencia del CCC en Argentina. En el primer apartado, abordamos dos principios que fundamentan este instituto, cuales son el principio de igualdad y el principio de solidaridad familiar. En el segundo, desplegamos algunos dilemas vinculados con la procedencia, medición y cuantificación de la CE.

En cuanto a los principios, teniendo la orientación dada en los Fundamentos del Anteproyecto de Código Civil y Comercial de la Nación Argentina (2014) referida a una ética de los/las vulnerables, pusimos el énfasis en tres preguntas desplegadas a partir de algunos aspectos, entre los que retomamos dos. En primer lugar, efectivamente es la CE un instituto que tiene la posibilidad de equilibrar relativamente situaciones de desigualdad real frente a la ruptura del matrimonio (o por el cese de la unión convivencial). Pero además, a través de la CE no solo el derecho puede equilibrar precariamente esa situación. Este instituto muestra (y demuestra) que es en la propia configuración de la comunidad matrimonial (o convivencial, según el caso) en la que se genera (de producirse) esa situación de desigualdad que ha sido naturalizada o incluso, normalizada. Y que frente a la interrupción (divorcio o cese de la unión convivencial), se hace contundentemente visible. Este es a nuestro entender el elemento político jurídico más potente de este instituto cuyas consecuencias en todo el sistema jurídico son inconmensurables.

En segundo lugar, con respecto a la ética de los/las vulnerables aplicada a esta figura, creemos que el meollo del término vulnerabilidad en el tercer uso que indicáramos, reside en las causas estructurales que la generan, es decir, se trata de un efecto social, económico, político y jurídico. Sea que se nazca en situación de vulnerabilidad, sea que se arribe a un estado de vulnerabilidad, lo que da contenido a esa situación es el marco contextual que lo produce (independientemente de la agencia que individual o colectivamente se posea). 
Lerussi, R y Scocozza, R.D. Elaboraciones jurisprudenciales en torno a la Compensación Económica en Argentina Derecho y Ciencias Sociales. Octubre 2018. Nº Pgs 93-112 ISNN 1852-2971. Instituto de Cultura Jurídica

Dicho en otros términos, la retórica de la vulnerabilidad no es sino una manera de situar el problema de la desigualdad en el debate ético político de una comunidad, cualquiera sea su escala (incluida la familia, el matrimonio, el grupo convivencial). En este sentido, es necesario entonces crear mecanismos estatales de igualación, es decir, que la comunidad acoja a quienes por razones estructurales están en situación de vulnerabilidad, diferente a situarlos en el lugar de víctimas a proteger. Desde el punto de vista del derecho, a veces acoger significa reconocer jurídicamente, es decir, dar existencia legal a lo que hasta entonces se mantuvo en la marginalidad. A veces acoger significa acentuar los recursos públicos para el cumplimiento de derechos y garantías. En ambos sentidos, entendemos, está hablándonos la incorporación del instituto de la CE con motivo de divorcio o del cese de la unión convivencial.

En cuanto al principio de solidaridad familiar indicamos, a través del fallo en estudio, la productividad que tiene la $\mathrm{CE}$ en la reconfiguración del mismo; esto intuimos tiene implicancias en todo el derecho de familia y en el sistema jurídico en su conjunto. Además, observamos la importancia en el marco del nuevo CCC de la valoración del trabajo doméstico y de cuidados no remunerados que realizan mayoritariamente las mujeres para la comunidad de vida, sea matrimonial o convivencial, no sólo en el presente sino frente a futuras contingencias que arriben en un quiere o finalización del vínculo. De este modo, se puede leer que la figura de la CE viene a visibilizar la importancia particularmente del trabajo no remunerado en la configuración de este principio. Configuración que tiene un carácter orientador no sólo en lo jurídico, sino a nivel social, político y afectivo.

En el segundo apartado, presentamos dilemas en torno a la cuantificación de la CE. Desarrollamos las respuestas dadas por los tribunales en fallos recientes que ensayan modos de cuantificación que, sin dudas, hablan de un instituto que requiere de maduración y apropiación en todas las instancias del derecho y a nivel social. Al ser una institución nueva, las discusiones en torno a su naturaleza (asistencial, resarcitoria, protectoria, sui generis) y a los presupuestos de procedencia, dan lugar a planteos que van desde lo creativo y novedoso, a lo que podemos calificar como audaz, temerario e incluso, en algunos casos, desconocedor de lo postulado por la legislación en relación a esta figura.

Simultáneamente, se advierte por parte de los tribunales a nivel nacional, una gran cautela al momento de determinar el reconocimiento de este rubro y fundamentar satisfactoriamente una cuantificación. Este es un punto delicado desde el punto de vista de la parte vulnerable que reclama un derecho a una $\mathrm{CE}$, en la mayoría de casos, mujeres. Además de aspectos asociados a la potencial vulneración de sus derechos económicos y patrimoniales, pueden 
Lerussi, R y Scocozza, R.D. Elaboraciones jurisprudenciales en torno a la Compensación Económica en Argentina Derecho y Ciencias Sociales. Octubre 2018. N Pgs 93-112 ISNN 1852-2971. Instituto de Cultura Jurídica

asomarse situaciones de violencia institucional (art. 6 b., Ley Nacional 26.485) por parte de jueces que ante la falta de acuerdo sobre el reconocimiento y la resolución de estos planteos, lo hagan por ejemplo sin una adecuada perspectiva de género. Esto porque las consecuencias a nivel práctico de uno y otro tipo de violencia son patentes: la violencia económica/patrimonial tiene como sujeto activo, en general, al ex marido/conviviente y la forma de revertirla es la demanda por CE ante el órgano jurisdiccional. Ahora, cuando es el órgano jurisdiccional el que no reconoce este derecho, no porque no se den sus presupuestos sino por aplicación de criterios civilistas y privatistas clásicos, previos al nuevo CCC, la violencia privada de índole económico patrimonial se torna en violencia institucional. Dicho en otros términos, violencia ejercida por órganos estatales cuya razón de existencia se fundamenta en la aplicación de la ley de acuerdo a los marcos constitucional y convencional vigentes. Decimos esto porque no es un dato menor que a poco más de dos años de vigencia de un instituto que ha tenido gran recepción en el derecho comparado, tengamos menos de una docena de fallos a nivel nacional que lo tratan desde distintos aspectos.

Como se desprende de la lectura de este artículo, los fallos citados dejan en evidencia que hay jurisdicciones que carecen por completo de decisiones jurisprudenciales que aborden el tema, sea para receptar el pedido y cuantificarlo, sea para fundamentar su rechazo. Sin pretensiones de cientificidad pero sí con la clara intuición que la práctica académica y privada nos dan, nos preguntamos preliminarmente si esta escasez de decisiones judiciales se debe a la ausencia de una adecuada perspectiva de género en el análisis de los casos concretos en los que la CE es solicitada ante la falta de acuerdo de quienes se separan o divorcian.

Luego de la reseña de este número reducido de fallos podemos afirmar que a más de dos años de la sanción del nuevo Código Civil aún resulta difícil identificar criterios o tendencias jurisprudenciales que caractericen adecuadamente el centro de gravedad (Pellegrini, 2017) de la CE mediante su acogimiento o rechazo. De allí la importancia y la urgencia de insistir en la profundización y argumentación garantistas respecto de la CE, dentro de los desafíos del nuevo CCC y en el marco del progresivo proceso de constitucionalización del derecho privado.

\section{Bibliografía}

Acciarri, H. A., (2015), Elementos de Análisis Económico del Derecho de Daños, Buenos Aires, Argentina: La Ley. 
Lerussi, R y Scocozza, R.D. Elaboraciones jurisprudenciales en torno a la Compensación Económica en Argentina Derecho y Ciencias Sociales. Octubre 2018. N Pgs 93-112 ISNN 1852-2971. Instituto de Cultura Jurídica

Belio Pascual, A. C., (2013), La pensión compensatoria, Valencia, España: Tirant lo Blanch, Valencia.

Constitución Nacional Argentina (CN) (1853; 1994), Argentina. Recuperada de http://bibliotecadigital.csjn.gov.ar/Constitucion-de-la-Nacion-Argentina-Publicaciondel-Bicent.pdf [16/08/2017].

De la Torre, N. y Pellegrini, M. V. (2016). Uniones convivenciales. En M. Herrera; A. Kemelmajer de Carlucci y N. Lloverás (Eds.). Tratado de Derecho de Familia, tomo V-A (pp. 385 - 396). Santa Fe, Argentina: Rubinzal - Culzoni.

El Hay, N. (2013). Derechos del personal de casas particulares a partir de abril de 2013. Revista de Derecho Laboral (2013 - 2), pp. 217-239.

Fundamentos del Anteproyecto de Código Civil y Comercial de la Nación Argentina (2014), Argentina. Recuperado de http://www.nuevocodigocivil.com/textos-oficiales-2/ [11/08/2017].

Herrera, M., Kemelmajer de Carlucci, A. y Lloverás, N. (Eds.), (2014), Tratado de Derecho de Familia según el Código Civil y Comercial de 2014, Buenos Aires, Argentina: Rubinzal Culzoni Editores.

Highton, E. (2015). Una etapa histórica: la mujer en el nuevo Código Civil y Comercial de la Nación. La Ley (año LXXIX, número 143, tomo 2015-D), pp. 5- 10.

Ley Nacional N²6.994 sobre Código Civil y Comercial de la Nación (2014), Argentina. Recuperada de http://www.infoleg.gob.ar/infolegInternet/anexos/235000239999/235975/norma.htm [10/08/2017].

Medina, G. (2012). Compensación económica en el Proyecto de Código. La Ley (1), cita online

AR/DOC/4860/2012. Recuperada de http://www.pensamientocivil.com.ar/doctrina/2639-compensaciones-economicascrisis-familiares-una-herramienta-equidad [17/11/2017].

Molina De Juan, M. (2012). Compensaciones económicas en el Proyecto. Una herramienta jurídica con perspectiva de género. Revista Derecho de Familia - RDF, pp. 57 - 193.

Molina De Juan, M. (2014). Alimentos y compensaciones económicas. En A. Kemelmajer y M. Molina de Juan (Dir.), Alimentos (pp. 299 - 346). Buenos Aires, Argentina: Rubinzal Culzoni.

Pautassi, L., (2007), El cuidado como cuestión social desde un enfoque de derechos. Santiago de Chile, Chile: CEPAL.

Pellegrini, M. V. (2014). Comentario a los artículos 441 a 442. En M. Herrera; A. Kemelmajer de Carlucci y N. Lloverás (Eds.), Tratado de Derecho de Familia según 
Lerussi, R y Scocozza, R.D. Elaboraciones jurisprudenciales en torno a la Compensación Económica en Argentina Derecho y Ciencias Sociales. Octubre 2018. N Pgs 93-112 ISNN 1852-2971. Instituto de Cultura Jurídica

el Código Civil y Comercial de 2014, tomo II (pp. 412 - 480). Buenos Aires, Argentina: Rubinzal Culzoni.

Pellegrini, M. V. (2017). Dos preguntas inquietantes en la compensación económica. $R C C y C$ (28), cita online AR/DOC/356/2017. Recuperado de http://thomsonreuterslatam.com/2017/05/dos-preguntas-inquietantes-en-lacompensacion-economica/ [17/11/2017].

Ojeda, R. (2013). Justificación de la ley 26.844. Revista de Derecho Laboral (2013 - 2), pp. 41-56.

Pérez Orozco, A., (2006), Perspectivas feministas en torno a la economía: el caso de los cuidados, Madrid, España: Consejo Económico y Social.

Silbaugh, K. (1996; 2012). Convirtiendo el trabajo en amor: el trabajo doméstico y el derecho. En N. Gherardi (Ed.), Justicia, género y trabajo (pp. 123 - 174). Buenos Aires, Argentina: Libraria / Red Alas.

Solari, N. (2014). Criterios de fijación de la prestación compensatoria. Revista de Derecho de Familia y de las Personas $\left(\mathrm{N}^{\mathrm{o}}\right.$ 27, junio, revista online sujeta a suscripción, referencia: AR/DOC/1556/2014) [01/07/2017].

Zelizer, V., (2009), La negociación de la intimidad, Buenos Aires, Argentina: FCE. 